\title{
Estado nutricional e práticas alimentares de idosos do Piauí: dados do Sistema de Vigilância Alimentar e Nutricional - SISVAN Web
}

\author{
Nutritional status and eating habits of elderly people in Piauí: available \\ data in SISVAN Web
}

\section{Estado nutricional y hábitos alimenticios de ancianos de Piauí: datos del SISVAN Web}

\author{
Roberto Lucas Moura Ruben Pereira ${ }^{1, a}$ \\ robertolmrp@hotmail.com | https://orcid.org/0000-0001-9258-0222 \\ Jéssica Pinheiro Mendes Sampaio ${ }^{2, b}$ \\ jessica_pinheiroms@hotmail.com | https://orcid.org/oooo-0002-1256-0772 \\ ${ }^{1}$ Centro Universitário INTA. Sobral, CE, Brasil. \\ 2 Universidade Federal do Piauí. Picos, PI, Brasil. \\ ${ }^{a}$ Graduando em Medicina pelo Centro Universitário INTA \\ ${ }^{\text {b }}$ Mestrado em Alimentos e Nutrição pela Universidade Federal do Piauí.
}

\section{Resumo}

A avaliação do estado nutricional dos idosos possibilita uma intervenção adequada, a fim de evitar ou minimizar agravos à saúde que comprometam a capacidade funcional dessas pessoas. Nessa perspectiva, $o$ trabalho apresentado neste artigo teve como objetivo verificar o estado nutricional e hábitos alimentares da população idosa do estado do Piaú. Foram utilizados dados de acesso público disponíveis no SISVAN Web referentes à avaliação nutricional realizada nos anos de 2014 a 2018. Observou-se aumento da prevalência de sobrepeso naquela população e uma diminuição do número de pessoas com baixo peso. Quando avaliados por sexo, as mulheres apresentaram maiores percentuais de excesso de peso em todos aqueles anos. O perfil do consumo alimentar mostrou que a maioria dos idosos consome alimentos in natura como frutas, verduras e feijão, e que, entre os que participaram da amostra, poucos fizeram uso de alimentos ultraprocessados. Por fim, cabe ressaltar a importância da avaliação do quadro de saúde da população como forma de subsídio para o planejamento de ações de saúde, dessa forma alocando melhor os recursos e possibilitando uma atuação eficaz dos que trabalham na área da saúde.

Palavras-chave: Envelhecimento; Vigilância nutricional; Saúde Pública; Sistemas de informação em saúde; Fatores de risco. 


\begin{abstract}
The evaluation of the nutritional status of the elderly allows the appropriate nutritional intervention avoiding or minimizing health problems that cause damage on their functional capacity. From this perspective, the study presented in this article aimed to verify the nutritional status and eating habits of the elderly population of the state of Piaú, Brazil. We used public access data available in the SISVAN Web regarding the nutritional evaluation carried out from 2014 to 2018. The data revealed an increase in the prevalence of overweight in that population and a reduction of the number of elderly with low weight. When evaluated by sex, women presented higher percentages of overweight in all those years. The food consumption profile showed that the majority of the eldery population consumes fresh foods such as fruits, vegetables and beans and only a few of participants of the sample consumed ultra-processed foods. Finally, the importance of the evaluation of the health of the population as a form of incentive to plan health actions is highlighted, so that the resources can be more competently allocated and the health care workers can act efficiently and effectively.
\end{abstract}

Keywords: Aging; Nutritional surveillance; Public Health; Health information systems; Risk factors.

\title{
Resumen
}

La evaluación del estado nutricional de las personas mayores permite una intervención nutricional adecuada evitando o minimizando los problemas de salud que comprometen su capacidad funcional. Desde esta perspectiva, el estudio presentado en este artículo tuvo como objetivo verificar el estado nutricional y los hábitos alimenticios de la población anciana del estado de Piauí, Brasil. Utilizamos los datos de acceso público disponibles en el SISVAN Web con respecto a la evaluación nutricional realizada desde los años 2014 hasta 2018. Se observó un aumento en la prevalencia del sobrepeso en laquella población y una disminución en el número de personas que tenían bajo peso. Cuando fueron evaluados por sexo, las mujeres presentaron porcentajes más altos de sobrepeso en todos aquellos años. El perfil del consumo de alimentos mostró que la mayoría de los ancianos consumía alimentos frescos como frutas, verduras y frijol, y pocos participantes de la muestra habían consumido alimentos ultraprocesados. Por fin, debe ser destacada la importancia de la evaluación de la salud de la población como una forma de incentivo para la planificación de acciones de salud, asignando así mejores recursos y posibilitando una actuación de los trabajadores de la salud eficiente y eficaz.

Palabras chave: Envejecimiento; Vigilancia nutricional; Salud Pública; Sistemas de informacíón en salud; Factores de riesgo.

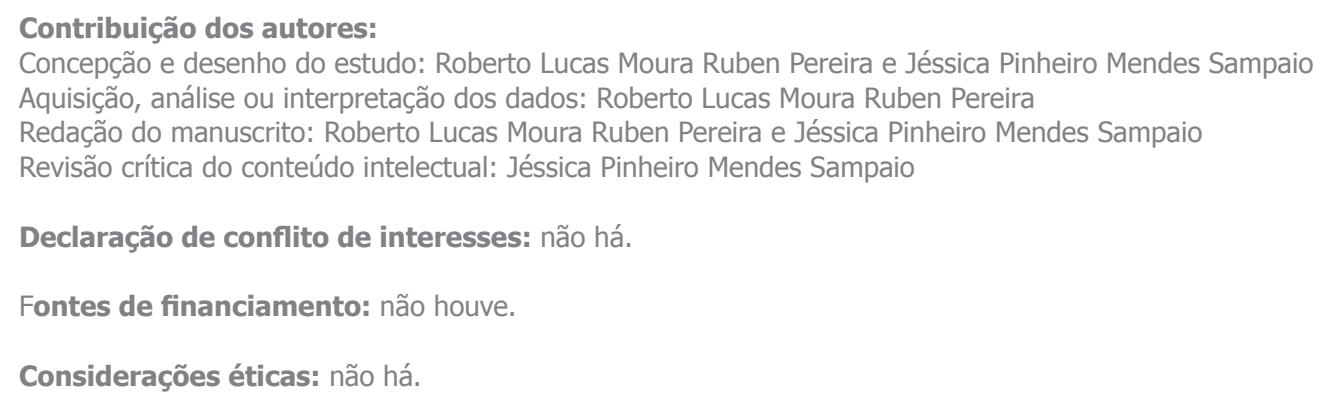




\section{Introdução}

Nos últimos anos houve um crescimento significativo da população idosa, devido a melhorias no nível socioeconômico e nos serviços de saúde, e estima-se que em 2025 o Brasil terá a sexta população de idosos do mundo, com cerca de 32 milhões de indivíduos nessa faixa etária ${ }^{1,2}$.

Com isso, aumentou-se a preocupação com esse grupo populacional, sendo necessário o conhecimento de todas as necessidades da terceira idade e o planejamento da atenção em saúde para garantir o acesso a serviços de saúde de qualidade³.

O envelhecimento é um processo múltiplo e desigual de comprometimento das funções do organismo e varia de indivíduo para indivíduo. Fatores como estilo de vida, condições socioeconômicas, presença de doenças crônicas e fatores psíquicos e emocionais interferem nesse processo e na qualidade de vida do idoso ${ }^{4,5}$.

Moraes, Moraes e Lima ${ }^{6}$ trazem em seu estudo a divisão do envelhecimento em dois aspectos: biológico e psíquico. O envelhecimento biológico é irreversível, causando vulnerabilidade do organismo, e é de natureza multifatorial, dependendo da programaçãogenética ealterações queocorrem no nível celular. Jáo envelhecimento psíquico ou amadurecimento depende do esforço pessoal na busca do autoconhecimento; a pessoa idosa tornase capaz de tolerar a dor ou a perda da independência biológica, e assim ele não é naturalmente progressivo.

O conjunto de alterações que ocorrem nesse momento da vida culmina na maior necessidade de ajuda, sendo este o principal fator que faz os idosos temerem a velhice, ou seja, a possibilidade de se tornarem dependentes de outras pessoas para exercerem suas atividades cotidianas ${ }^{1,7}$.

Dessa forma, torna-se necessário não apenas aumentar a expectativa de vida, mas também a melhoria das condições para uma velhice ativa e saudável, motivos pelos quais se destaca a importância das políticas públicas de saúde ${ }^{8}$.

A avaliação do estado nutricional (EN) do idoso possibilita a intervenção em sua nutrição adequada, evitando ou minimizando agravos à saúde que comprometam a capacidade funcional. E, entre os instrumentos utilizados, pode-se citar a Mini-Avaliação Nutricional (MAN), o Índice de Massa Corpórea (IMC) e a Circunferência da Panturrilha (CP) 9 .

E como forma de monitoramento da saúde da população, o Sistema de Vigilância Alimentar e Nutricional (SISVAN) corresponde a um sistema de coleta, processamento e análise contínua dos dados, possibilitando diagnóstico da situação nutricional, assim como os seus fatores determinantes. O SISVAN disponibiliza dados de grupos específicos da população, como idosos, crianças, o que permite a identificação dos grupos de risco e planejamento de ações de saúde ${ }^{10}$.

Nessa perspectiva, o presente trabalho tem como objetivo verificar o estado nutricional e as práticas alimentares da população idosa do estado do Piauí.

\section{Estratégia metodológica}

Trata-se de um estudo retrospectivo realizado por meio de dados remissivos, no qual foi avaliado o estado nutricional dos idosos residentes no estado do Piaú, assim como os seus hábitos alimentares. Todos os dados desta pesquisa foram obtidos na plataforma SISVAN Web e estão disponíveis para consulta livre via web no seguinte endereço eletrônico: http://sisaps.saude.gov.br/sisvan/relatoriopublico/index.

O estado do Piaú, de acordo com o último censo (2010), possui 3.118.360 milhões de habitantes, com densidade demográfica de 12,40 hab $/ \mathrm{km}^{2}$, está dividido em quatro mesoregiões e 15 microrregiões, constituídas de 224 municípios ${ }^{11}$. Quanto às regiões de saúde, estão divididas em dez: Carnaubais, Chapada das Mangabeiras, Cocais, Entre Rios, Planície Litorânea, Serra da Capivara, Tabuleiros do Alto Parnaíba, Vale do Canindé, Vale do Rio Guaribas, Vale do Sambito e Vale dos Rios Piauí e Itaueras. 
Os critérios de inclusão consistiram em dados referentes aos idosos acompanhados e registrados no SISVAN Web no estado do Piauí ao longo dos anos de 2014 a 2018 de todas as regiões de cobertura e escolaridade; para análise do perfil alimentar, foram coletados os dados do ano mais recente desse sistema. A escolha da faixa etária se deu devido ao significativo crescimento da população idosa e da relação entre o estado nutricional e a qualidade de vida desse grupo.

Os dados utilizados são provenientes de unidades básicas de saúde e constituem-se da classificação do Índice de Massa Corporal (IMC) e da avaliação de marcadores do consumo alimentar realizados durante o atendimento individual nas respectivas unidades.

A obtenção das medidas antropométricas para avaliação do estado nutricional foi realizada segundo a metodologia preconizada no manual do SISVAN ${ }^{12}$, com a seguinte classificação:

- Valores de IMC menor ou igual a 22: idoso com baixo peso;

- Valores de IMC maior que 22 e menor que 27: idoso com peso adequado (eutrófico);

- Valores de IMC maior ou igual a 27: idoso com sobrepeso.

As informações do consumo alimentar foram coletadas através do Formulário de Marcadores do Consumo Alimentar com questões sobre o consumo do dia anterior, no qual o indivíduo responde se consumiu ou não os alimentos listados. Tem como objetivo caracterizar a alimentação atual e verificar a adoção de hábitos inadequados.

Os dados obtidos foram organizados em tabelas e gráficos em percentuais e números absolutos com o auxílio do software Excelß).

\section{Resultados}

Na Tabela 1 estão os valores de IMC dos idosos cadastrados e acompanhados durante os anos de 2014 a 2018.

Tabela 1-Estado nutricional de idosos cadastrados no SISVAN no estado do Piaví, 2014-2018

\begin{tabular}{lccccc}
\hline Estado nutricional & $\mathbf{2 0 1 4}$ & $\mathbf{2 0 1 5}$ & $\mathbf{2 0 1 6}$ & $\mathbf{2 0 1 7}$ & $\mathbf{2 0 1 8}$ \\
\hline Baixo peso & $19,37 \%$ & $17,14 \%$ & $16,69 \%$ & $15,46 \%$ & $14,55 \%$ \\
Eutrófico & $41,22 \%$ & $41,61 \%$ & $42,13 \%$ & $42,24 \%$ & $41,05 \%$ \\
Sobrepeso & $39,41 \%$ & $41,25 \%$ & $41,18 \%$ & $42,30 \%$ & $44,41 \%$ \\
Total de idosos & 4.260 & 30.221 & 45.709 & 48.758 & 52.218 \\
\hline
\end{tabular}

Fonte: Os autores (2019) a partir de SISVAN (2019).

Com relação à avaliação do estado nutricional, houve diminuição dos percentuais de baixo peso no decorrer dos anos avaliados, sendo de 19,37\% em 2014 e passando para 14,55\% em 2018. Em contrapartida, foi registrado aumento de sobrepeso no grupo em estudo, do qual quase metade $(44,41 \%)$ dos idosos acompanhados em 2018 estavam com valor de IMC acima do recomendado. 
Na Figura 1, estão demonstrados os percentuais encontrados sobre o estado nutricional de acordo com o sexo.

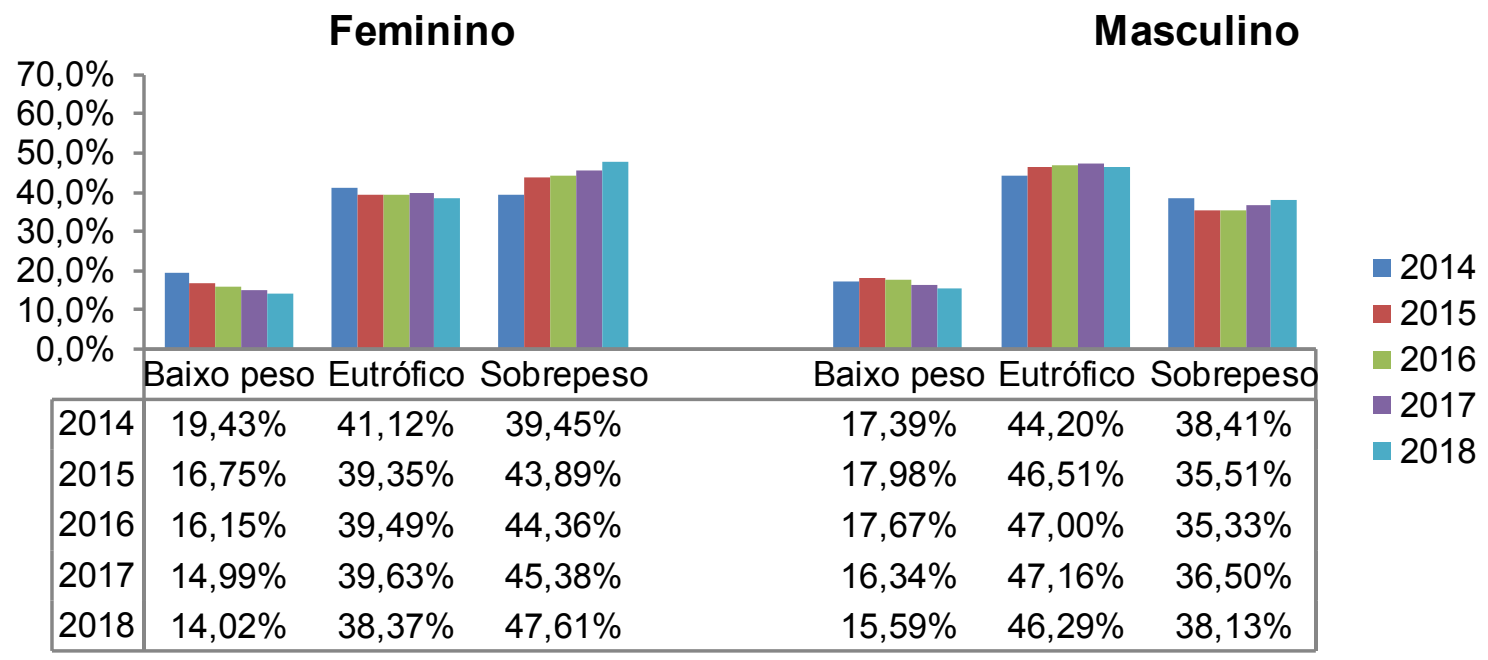

Figura 1 - Estado nutricional de idosos cadastrados no SISVAN no estado do Piauí, segundo o sexo, 2014-2018 Fonte: Os autores (2019).

Quando comparados os dois grupos, observa-se que a cada ano aumenta o percentual de idosas com sobrepeso e, em todos os anos, ele é maior do que o dos homens idosos, chegando a 47,61\% no último ano, maior que o do valor geral. E, da mesma forma, percentuais maiores de peso adequado foram encontrados entre os homens, ou seja, o desvio de estado nutricional, seja relacionado ao excesso ou ao déficit, foi maior entre as mulheres.

Moraes $^{13}$ ressalta que o Índice de Massa Corporal (IMC) é um método simples e que se correlaciona bem com outros métodos de avaliação nutricional. O autor salienta também que, no que se refere à população idosa, conservar o peso adequado caracteriza umas das medidas adequadas para a prevenção de fraturas e manutenção da independência.

Na Tabela 2, estão demonstrados os dados sobre o consumo alimentar do último ano avaliado no presente estudo, que foi escolhido por ser o com maior número de idosos cadastrados (52.218). No entanto, cabe salientar que a amostra foi constituída por 1.681 idosos com relatório do consumo alimentar cadastrado.

Tabela 2 - Número e porcentagem de idosos segundo os hábitos e consumo alimentar no dia anterior, Piauí, 2018

(continua)

\begin{tabular}{lcc}
\hline Variável & $\mathbf{N}$ & \% \\
\hline Feijão & & \\
Sim & 1.473 & $88 \%$ \\
Não & 208 & $12 \%$ \\
Frutas frescas & & \\
Sim & 1.253 & $75 \%$ \\
Não & 428 & $25 \%$ \\
Verduras e/ou legumes & & \\
Sim & 1.067 & $63 \%$ \\
Não & 614 & $37 \%$
\end{tabular}




\begin{tabular}{|c|c|c|}
\hline Variável & $\mathbf{N}$ & $\%$ \\
\hline \multicolumn{3}{|c|}{ Hambúrguer e/ou embutidos } \\
\hline Sim & 218 & $13 \%$ \\
\hline Não & 1.463 & $87 \%$ \\
\hline \multicolumn{3}{|c|}{$\begin{array}{l}\text { Bebidas adoçadas (refrigerante, suco de caixinha, suco em pó, } \\
\text { água de coco de caixinha, xaropes de guaraná/groselha, suco } \\
\text { de fruta com adição de açúcar) }\end{array}$} \\
\hline Sim & 484 & $29 \%$ \\
\hline Não & 1.197 & $71 \%$ \\
\hline \multicolumn{3}{|c|}{ Macarrão instantâneo, salgadinhos de pacote ou biscoitos salgados } \\
\hline Sim & 468 & $28 \%$ \\
\hline Não & 1.213 & $72 \%$ \\
\hline \multicolumn{3}{|c|}{$\begin{array}{l}\text { Biscoito recheado, doces ou guloseimas (balas, pirulitos, chiclete, } \\
\text { caramelo, gelatina) }\end{array}$} \\
\hline Sim & 269 & $16 \%$ \\
\hline Não & 1.412 & $84 \%$ \\
\hline \multicolumn{3}{|c|}{$\begin{array}{l}\text { Costume de realizar as refeições assistindo à TV, mexendo no } \\
\text { computador e/ou celular? }\end{array}$} \\
\hline Sim & 387 & $24 \%$ \\
\hline Não & 1.294 & $76 \%$ \\
\hline \multicolumn{3}{|c|}{ Hábito de realizar no mínimo as três refeições principais do dia } \\
\hline Sim & 43 & $3 \%$ \\
\hline Não & 1.638 & $97 \%$ \\
\hline
\end{tabular}

Fonte: Os autores (2019) a partir de SISVAN (2019).

Como forma de monitoramento do estado de saúde dos indivíduos, durante o atendimento nas unidades de saúde é aplicado, além de outras estratégias, um questionário sobre o consumo alimentar, no qual as perguntas variam de acordo com o grupo etário em que o paciente se enquadra e para os idosos são relacionadas ao consumo do dia anterior referente à ingestão de grupo de alimentos (verduras ou legumes), de alimentos específicos (embutidos, bebidas adoçadas) e também aos hábitos de realização das três principais refeições.

Os maiores percentuais de afirmação de consumo foram relatados para alimentos in natura ou minimamente processados: $88 \%$ dos idosos afirmaram ter consumido feijão, $75 \%$ frutas frescas e $63 \%$ da amostra referiu a ingestão de verduras e/ou legumes. Já com relação a alimentos que devem ser evitados por serem processados ou ultraprocessados, observou-se o consumo de bebidas adoçadas por $29 \%$ dos avaliados e de macarrão instantâneo, salgadinho de pacote ou biscoito salgado por 28\% deles.

No que diz respeito aos hábitos de consumo, duas informações preocupantes foram que $97 \%$ dos idosos avaliados referiram não terem realizado, no mínimo, as três principais refeições do dia e $24 \%$ afirmaram realizar as refeições assistindo à televisão ou mexendo no celular, hábitos esses que se relacionam e contribuem para os desvios encontrados na avaliação do estado nutricional da amostra.

\section{Discussão}

O envelhecimento rápido da população traz inúmeras consequências, tanto para o indivíduo, família e comunidade, como também para as redes de atenção à saúde e planejamento das estratégias de intervenção e prevenção. O que se busca é um envelhecimento saudável, com manutenção da qualidade de vida e independência do idoso, o que reflete na sua autonomia para tarefas do dia a dia e para escolhas como a do preparo e consumo alimentar. 
Nesse contexto o estado nutricional assume um importante papel, e, entre as ferramentas disponíveis para sua avaliação, as medidas antropométricas apresentam-se como as mais utilizadas, com destaque para o emprego do IMC, que apesar de não diferenciar massa magra de massa gorda, possibilita a classificação em graus de nutrição e permite boa correlação com graus de morbimortalidade ${ }^{14}$.

Os valores de sobrepeso encontrados entre os idosos no Piauí estão de acordo com outros estudos sobre o tema ${ }^{15-16}$, nos quais se observaram percentuais de $45 \%$ e 42,8\% de excesso de peso nos estados de Minas Gerais e Ceará. Isso se explica pelo processo natural de envelhecimento, ocorrendo mudanças na composição corporal com o aumento progressivo de gordura e a sua redistribuição, sendo o maior acúmulo na região abdominal.

O aumento do excesso de peso e da obesidade vem acontecendo no Brasil e no mundo. Em 2016, o Ministério da Saúde divulgou o Vigitel (Vigilância de Fatores de Risco para Doenças Crônicas por Inquérito Telefônico), no qual foi registrado que mais da metade $(52,5 \%)$ da população brasileira está acima do seu peso ideal ${ }^{17}$.

Com relação à diferença dos percentuais de excesso de peso quando analisados por sexo, o maior número de mulheres com sobrepeso também foi encontrado em outra pesquisa ${ }^{14}$, e pode advir de uma explicação de ordem fisiológica. Não só as mulheres acumulam maior gordura que os homens e a perdem em idades mais tardias, como também pode haver contribuição da menopausa acompanhada do aumento de peso e adiposidade.

Cabe ressaltar que o excesso de peso está relacionado com o aparecimento de Doenças Crônicas não Transmissíveis (DCNT) e, dessa forma, os idosos com peso acima do recomendado têm risco de morbimortaliade por patologias cardiovasculares e diabetes melitus ${ }^{15,18}$.

É preciso atenção também para o baixo peso, que embora tenha percentuais menores que o sobrepeso, é um quadro bastante incidente entre os idosos e que contribui para a mortalidade ${ }^{14}$. A desnutrição reduz a qualidade de vida, pois entre outros fatores, leva à maior diminuição da massa muscular e redução da capacidade de ação, o que contribui para a incapacidade funcional, deixando o idoso dependente de um cuidador ${ }^{19}$.

Estudos que avaliaram o estado nutricional de idosos encontraram percentuais de baixo peso de $13,8 \%^{20}$ e de $10 \%^{21}$, resultados condizentes com os do presente estudo. Além disso, observa-se que, quando são avaliados idosos institucionalizados, é encontrado maior percentual de déficit de IMC, totalizando 30,97\% em $1994^{22}$.

Os dados referentes ao consumo alimentar trazem informações sobre alimentos ingeridos no dia anterior, tendo como principal enfoque avaliar o consumo qualitativo da alimentação, ou seja, se há a inserção de frutas e vegetais, e também se há consumo de alimentos inadequados.

O Guia Alimentar para a População Brasileira ${ }^{23}$ foi reformulado em 2014 e não traz referências quantitativas de consumo e/ou nutrientes, mas sim os princípios básicos da alimentação e uma 'regra ouro' segundo a qual deve ser dada preferência sempre a alimentos in natura ou minimamente processados e devem ser feitas preparações culinárias a alimentos ultraprocessados.

Nessa perspectiva, o perfil de consumo dos idosos avaliados, no estudo aqui apresentado, estava em sua maioria adequado, dada a ingestão de feijão, frutas frescas e verduras e/ou legumes. O feijão constitui junto com o arroz o prato básico da alimentação do brasileiro e é um hábito muito forte principalmente nas regiões Norte e Nordeste do país, o que explica o alto consumo pelo grupo; essa combinação melhora a qualidade da proteína ingerida, pois supre os aminoácidos limitantes.

Um estudo realizado com 416 idosos em Goiânia, Goiás ${ }^{24}$, encontrou prevalência de consumo diário de frutas em $44 \%$ dos entrevistados, de verduras em 39,7\% e de legumes em $32,5 \%^{24}$, valores menores que os encontrados no presente estudo; porém, cabe ressaltar que esses valores observados são de um consumo diário e não apenas do dia anterior como indagado na avaliação do formulário do SISVAN. A ingestão de 
verduras e frutas deve ser sempre incentivada por conterem vitaminas, minerais e compostos antioxidantes que atuam no combate dos radicais livres.

No presente estudo, o consumo de alimentos ultraprocessados apresentou valores baixos entre os idosos cadastrados, o que foi observado também em pesquisa realizada com 1.426 idosos em Pelotas, Rio Grande do $\mathrm{Sul}^{25}$, o que é um fator positivo, uma vez que esses alimentos possuem elevado teor calórico e são pobres de nutrientes. Os autores ressaltaram que esse baixo consumo pode estar relacionado à formação dos hábitos alimentares dos idosos, ocorrida em época cuja oferta e consequentemente o consumo desses produtos eram menores, ou mesmo ao entendimento popular de que esses alimentos são menos digestivos e inapropriados após uma determinada idade.

Já o fato de a maioria da amostra deste estudo não ter realizado, pelo menos, as três principais refeições no dia anterior é preocupante, uma vez que o consumo de um menor número de refeições está associado ao consumo de uma dieta de menor qualidade e essa ingestão inadequada pode levar à perda de peso e desnutrição. Esse fato pode estar associado a vários fatores como as condições socioeconômicas, a presença de patologias que dificultam a ingestão alimentar e até mesmo ao fato de não haver uma companhia durante as refeições ${ }^{25}$.

\section{Conclusões}

Os resultados mostraram que um alto percentual dos idosos da amostra deste estudo encontra-se com sobrepeso, e que esse percentual é ainda maior quando se trata das mulheres. O perfil do consumo alimentar mostrou que a maioria desses idosos consome alimentos in natura como frutas, verduras e feijão e poucos ingerem alimentos ultraprocessados.

O presente trabalho foi de grande contribuição por analisar o perfil de idosos atendidos de na rede pública, o que é de suma importância para o planejamento de ações de saúde, uma vez que grande parte dos indivíduos cadastrados no SISVAN Web está em atendimento por já possuir uma patologia associada aos problemas aqui abordados. Dessa forma, os Sistemas de Informação em Saúde também são de grande importância para melhor alocação dos recursos e atuação de forma eficiente e eficaz.

\section{Referências}

1. Ferreira OG, Maciel SC, Silva AO, Santos WS, Moreira MASP. O envelhecimento ativo sob o olhar de idosos funcionalmente independentes. Rev Esc Enferm USP. 2010;44(4):1065-9.

2. Vasconcelos AMN, Gomes MMF. Transição demográfica: a experiência brasileira. Epidemiol Serv Saúde. 2012;21(4):539-48.

3. Pilger $\mathrm{C}$, Menos MH, Mathias TAF. Características sociodemográficas e de saúde de idosos: contribuições para os serviços de saúde. Rev Latino-Am Enferm. 2011;19(5):1-9.

4. Fechine BRM, Trompieiri N. O processo de envelhecimento: as principais alterações que acontecem com o idoso com o passar dos anos. InterScience Place. 2012;7(1):106-32.

5. Garbin CAS, Sumida DH, Moimaz SAS, Prado RL, Silva, MM. O envelhecimento na perspectiva do cuidador do idoso. Ciênc Saúde Coletiva. 2010;15(6):2941-8.

6. Moraes EM, Moraes FL, Lima SPP. Características biológicas e psicológicas de envelhecimento. Rev Med Minas Gerais. 2010;20(1):67-73.

7. Freitas MC, Queiroz TA, Sousa JAV. O significado da velhice e da experiência de envelhecer para os idosos. Rev Esc Enferm USP. 2010;44(2):407-12.

8. Ciosask SI, Braz E, Costa MFBNA, Nakano NGR, Rodrigues J, Alencar RA, et al. Senescência e sensibilidade: novo paradigma na Atenção Básica de Saúde. Rev Esc Enferm USP. 2012;45(Esp.2):1763-8.

9. Martin FG, Nebuloni CC, Najas MS. Correlação entre estado nutricional e força de preensão palmar em idosos. Rev Bras Geriatr Gerontol. 2012;15(3):493-504. 
10. Camilo SMB, Camilo GBC, Toledo GC, Camilo Júnior RD, Toledo CC. Vigilância nutricional no Brasil: criação e implementação do SISVAN. Rev APS. 2011;14(12):224-8.

11. IBGE. Panorama estado do Piauí [Internet]. 2017 [citado 30 out 2018]. Disponível em https://cidades. ibge.gov.br/brasil/pi/panorama.

12. Ministério da Saúde (BR). Secretaria de Atenção à Saúde. Departamento de Atenção Básica. Orientações para a coleta e análise de dados antropométricos em serviços de saúde: Norma Técnica do Sistema de Vigilância Alimentar e Nutricional: SISVAN. Brasília; 2011.

13. Moraes EM. Atenção à saúde do idoso: aspectos conceituais. Brasília: Organização Pan-Americana da Saúde; 2012. 98 p.

14. Pereira IFS, Spyrides MHC, Andrade MLB. Estado nutricional de idosos no Brasil: uma abordagem multinível. Cad Saúde Pública. 2016;32(5):1-12.

15. Nascimento CM, Ribeiro AQ, Cotta RMM, Acurcio FA, Peixoto SV, Priore SE et al. Estado nutricional e fatores associados em idosos no município de Viçosa, Minas Gerais, Brasil. Cad Saúde Pública. 2011;12(27):2409-18.

16. Pinheiro WL, Coelho Filho JM. Perfil dos idosos usuários das academias ao ar livre para a terceira idade. Rev Bras Promoç Saúde. 2017;30(1):93-101. Ministério da Saúde (BR). Secretaria de Vigilância em Saúde. Departamento de Vigilância de Doenças e Agravos não Transmissíveis e Promoção da Saúde. Vigitel Brasil 2016: vigilância de fatores de risco e proteção para doenças crônicas por inquérito telefônico: estimativas sobre frequência e distribuição sociodemográfica de fatores de risco e proteção para doenças crônicas nas capitais dos 26 estados brasileiros e no Distrito Federal em 2016. Brasília; 2017.

17. Neumann B, Conde SR, Lemos JRN, Moreira TR. Associação entre o estado nutricional e a prevalência de doenças crônicas não transmissíveis em idosos residentes no município de Roca Sales-RS. Revista Brasileira de Ciências do Envelhecimento Humano. 2014; 11(2):166-177.

18. Sousa KT, Mesquita LAS, Pereira LA, Azevedo CM. Baixo peso e dependência funcional em idosos institucionalizados de Uberlândia (MG), Brasil. Ciênc Saúde Coletiva. 2014;19(8):3513-20.

19. Sass A, Marcon SS. Comparação de medidas antropométricas de idosos residentes em área urbana no sul do Brasil, segundo sexo e faixa etária. Rev Bras Geriatr Gerontol. 2015;18(2):361-72.

20. Salmaso FV, Vigário PS, Mendonça LMC, Madeira M, Netto LV, Guimarães MR et al. Análise de idosos ambulatoriais quanto ao estado nutricional, sarcopenia, função renal e densidade óssea. Arq Bras Endocrinol Metabol. 2014;58(3):226-31.

21. Azevedo EAM, Lopes HG, Maia AHS, Lima VT, Nunes VMA, Alchieri JC. Avaliação nutricional de idosos residentes em instituições filantrópicas. J Health Sci Inst. 2014; 32(3):260-64.

22. Ministério da Saúde (BR). Secretaria de Atenção à Saúde. Departamento de Atenção Básica. Guia alimentar para a população brasileira. 2 ed. Brasília; 2014.

23. Silveira EA, Martins BB, Abreu LRS, Cardoso CKS. Baixo consumo de frutas, verduras e legumes: fatores associados em idosos em capital no centro-oeste do Brasil. Ciênc Saúde Coletiva. 2015;20(12):3689-99.

24. Gomes AP, Soares ALG, Gonçalves H. Baixa qualidade da dieta de idosos: estudo de base populacional no sul do Brasil. Ciênc Saúde Coletiva. 2016;21(11):3417-3428. 